\title{
DEEP LEARNING FOR AGE GROUP CLASSIFICATION SYSTEM
}

\author{
Karthick R, \\ Department of Electronics and Communication Engineering, \\ Sethu Institute of Technology, \\ Madurai, India \\ karthickkiwi@gmail.com
}

\begin{abstract}
Age Group Classification (AGC) is a difficult task due to variations in human genders, expression, races, poses and so on. The age group analysis is used in multimedia forensic investigation for crime scenes. In this study, an efficient method for AGC is presented. AGC system uses mainly two stage; preprocessing and classification. The preprocessing stage consists of face region detection, gamma correction, Difference Of Gaussian (DOG) filter and normalization. Then the preprocessed images are given to Visual Geometric Group (VGG) 16. The convolution and max-pooling layers in VGG 16 architecture is used to resize the image. The REctified Linear Unit (RELU) is used as an activation function in each convolution and max-pooling layer. The sigmoid layer is used for the AGC into adolescence, adult and senior adult. The system uses MORPH database for the performance evaluation. AGC system produces the classification accuracy of over $90 \%$ for all age groups using VGG16 architecture.
\end{abstract}

Keywords: Age group classification, neural network, VGG16, MORPH database

\section{INTRODUCTION}

Classification of age group is described in [1] using facial images. The active appearance model is used to extract the facial image features. The feature dimensions are reduced by principal component analysis. The Support Vector Machine (SVM) Classifier with radial basis function kernel is used for classification. Curvelet features based human age-group classification is presented in [2]. The input finger print images are extracted by curvelet transform. The $k$-nearest neighbor is used for classification.

Age classification using facial images based on geometric features is discussed in [3]. At first, the geometric features are used to extract the input facial images. The neural network classifier is used for classification. Facial age classification using local age group modeling is discussed in [4]. The input facial images are extracted by Local Binary Patterns (LBP). The classification is made by Euclidean distance classifier.

SVM based frontal facial image classification is described in [5]. Initially, the input facial images are converted into grayscale image. The edges are detected by histogram equivalent method. The features extracted by haar wavelet transform SVM classifier is used for classification of facial images. Age information based gender classification is discussed in [6]. At first, the features are extracted by LBP and geometric features. The Euclidean distance of the SVM classifier is used for the classification.

SVM based AGC using facial images is described in [7]. At first, the face images are normalized. Surface projection features are used for the feature 
extraction. The features are reduced by principal component analysis and spectral regression. The facial images are classified by SVM classifier. AGC using gradient LBP is described in [8]. The local texture descriptor feature is used to extract the input image features. Gradient LBP is used for classification.

Human AGC using facial edges and correlation fractal dimension is described in [9]. Initially, the original input images are cropped and converted into grayscale. The edges are detected by canny edge operator. The fractal dimension values are estimated. The classification is made by local diagonal pattern. Classification of age group in various lighting conditions is described in [10]. The two-dimensional direct linear discriminant analysis and is used to extract the input image features. The linear discriminant analysis and principal component analysis is used for classification.

Facial feature based age classification is discussed in [11]. The wrinkle features and geometric features are used for feature extraction. The backpropagation neural network is used for classification. Human AGC using multiclass SVM is described in [12]. The Gabor filter is used to analyze the facial skin changes. Wrinkle analysis defect is used for the identifying age profession. The multiclass SVM is used for classification.

AGC using facial images using histogram oriented gradients is discussed in [13]. The histogram oriented gradients is used for feature extraction. Probabilistic neural network is used for classification. Artificial neural network based age classification using signals is described in [14]. The input signals are extracted by mean absolute value, variance, root mean square, zero crossing, slope sign change, median frequency and standard deviation. The classification is made by artificial neural network.

In this study, AGC system using VGG16 architecture is presented. The organization of paper is as follows: Methods and materials used in AGC system is discussed in section 2. Results and discussion of AGC system is described in section 3. The final section concludes the AGC system using VGG16 architecture.

\section{METHODS AND MATERIALS}

In this study, VGG16 model is utilized for AGC system. Figure 1 shows the workflow of AGC using preprocessing and classification stages. Initially, the face region is detected, and then gamma correction, DOG filtering and normalization are employed to preprocess the image to get higher accuracy. The preprocessed images are given to VGG16 architecture. The VGG16 classifies the age into adolescence, adult and senior adult groups.

\section{A. Preprocessing of Facial images}

The preprocessing is the essential stage for AGC system. In this study the preprocessing is made by face region detection, gamma correction, DOG filtering and normalization [15]. The facial region is detected because the MORPH database contains some background information so in order to remove the background information the face region is detected.

The overall brightness of the image is controlled by gamma correction. The dark and bleached out images are not properly corrected. The color reproduction needs the gamma correction. It also changes the ratio of red to green to blue. DOG filter is similar to different box filters and Gaussian Laplacian function. DOG filter is a band pass filter that removes all spatial frequency that present in the original gray scale image. 


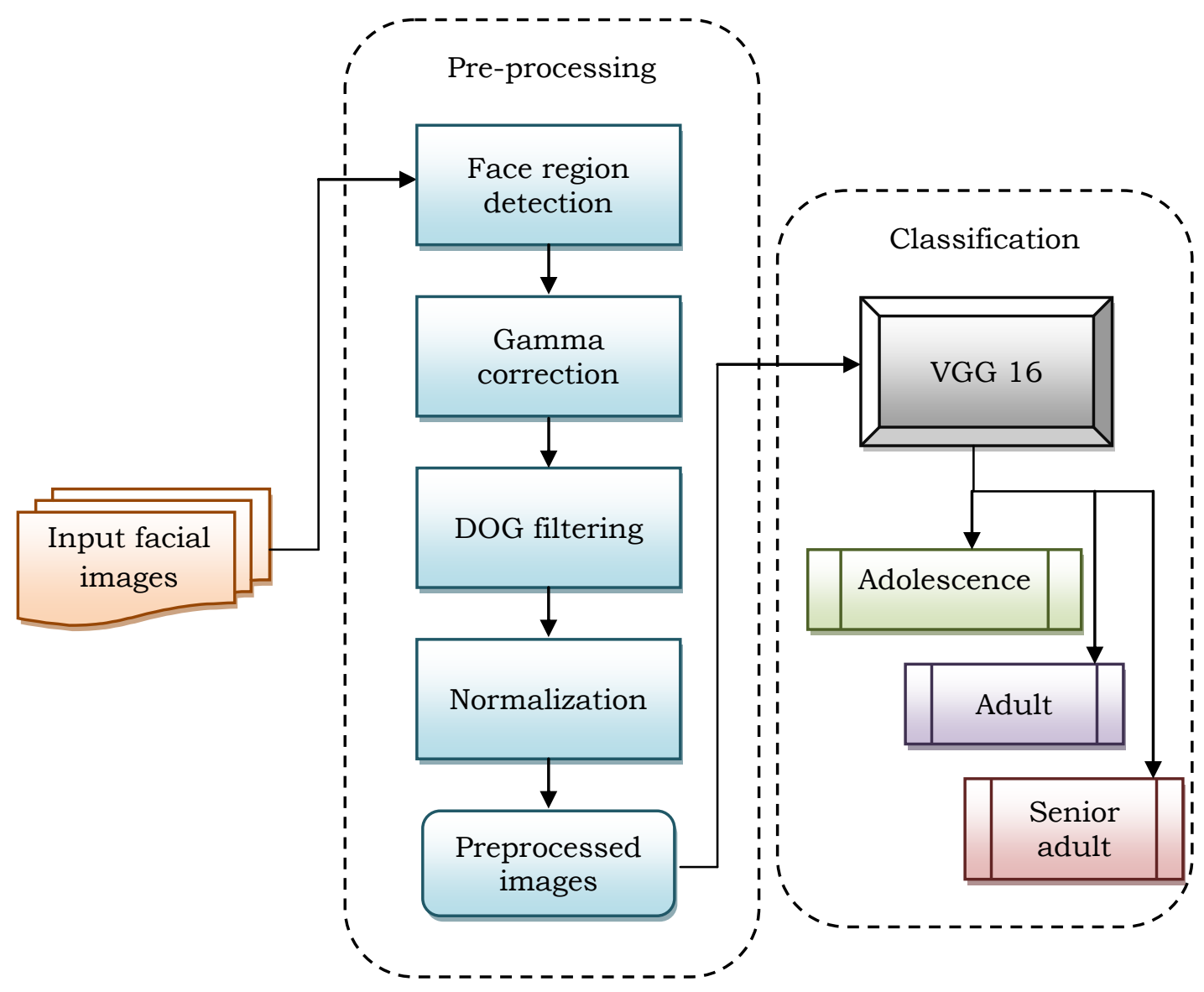

Fig. 1 Overall workflow of AGC system

The robust measures are standardized and rescaled by the image intensity. The robust estimator is used due to signals which have the large values produced by nostrils, highlights, garbage at the image border, nostrils, and so on. In this study the face regions are detected to remove the background surface, and then the gamma correction is made to control the overall brightness. After that, DOG filter is applied to remove the spatial frequency which is present in the gray scale image. Finally the normalization is made to rescale and standardize the image intensity.

\section{B. VGG16 Classification}

VGG16 architecture consists of five blocks with convolution and maxpooling layers. The first two blocks consist of two convolution layers and last three blocks consist of three convolution layer followed by max-pooling layer. ReLU activation function in used in as activation function of VGG16. It is used in each layer of convolution to predict the better output. Dense layer in VGG 16 is used to transfer the neurons from input to output channels. Figure 2 shows the VGG 16 architecture for age group classification. Sigmoid layer is used for classification. Sigmoid is a logistic function with "S" shape. The output of sigmoid is smoother than the input. In this study, the preprocessed facial images are given to VGG16 for classification. 


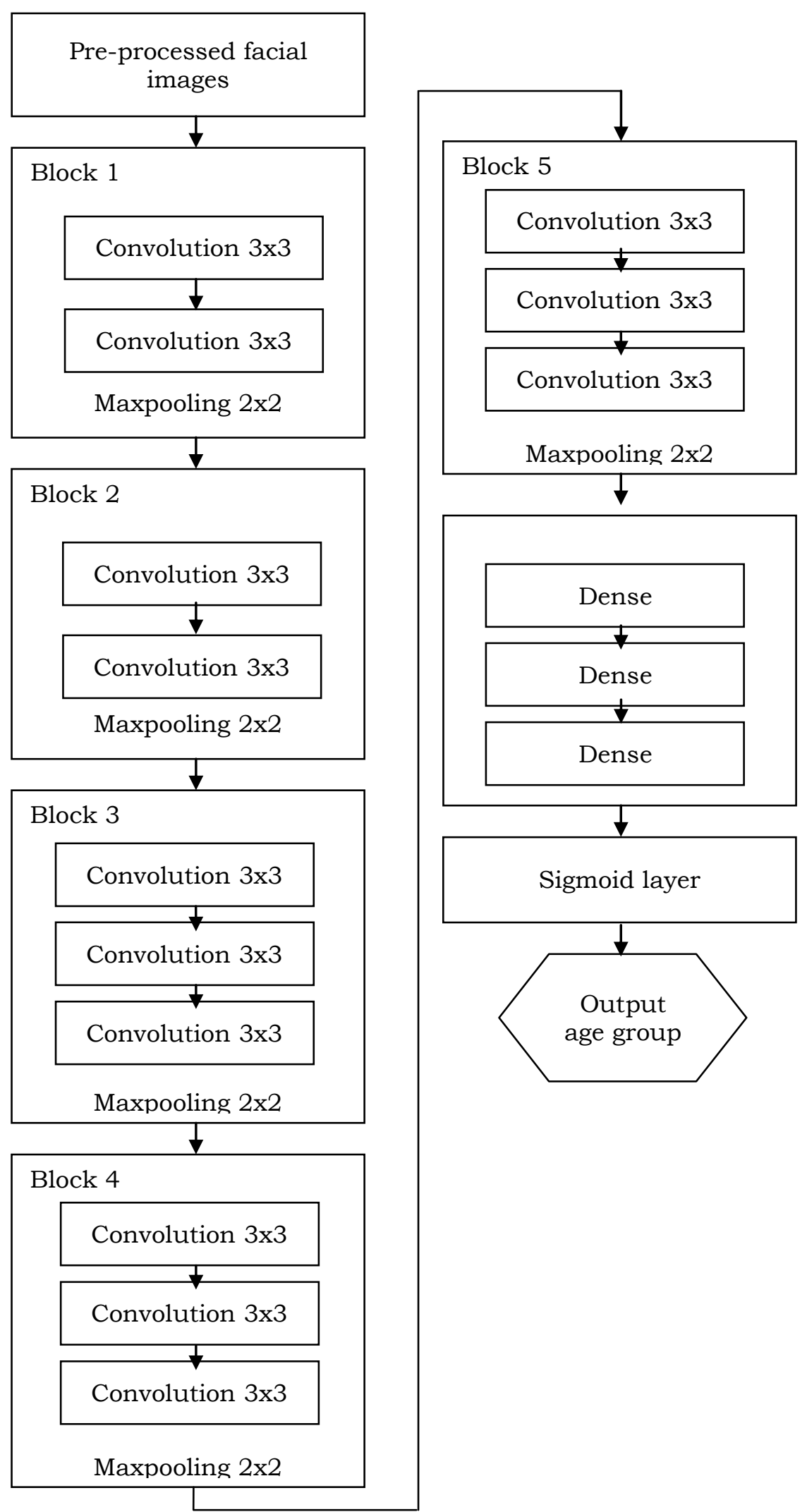

Fig. 2 VGG16 system for AGC 


\section{RESULTS AND DISCUSSION}

The GAC system uses MORPH database for performance evaluation. More information about MORPH database can be found in [16]. Table 1 shows the number of images available in each category.

TABLE 1 MORPH Database images in each category

\begin{tabular}{|c|c|c|}
\hline Age Group & Age Intervals & Number of images \\
\hline Senior Adult & $>59$ & 334 \\
\hline Adolescence & $13-19$ & 5377 \\
\hline Adult & $20-59$ & 49423 \\
\hline
\end{tabular}

From the database, only 250 images from senior adult and 500 images from other two categories are considered for performance evaluation. The images are randomly selected. Before classification, the images are preprocessed to remove background and illumination variance. Figure 3 shows the preprocessed images.
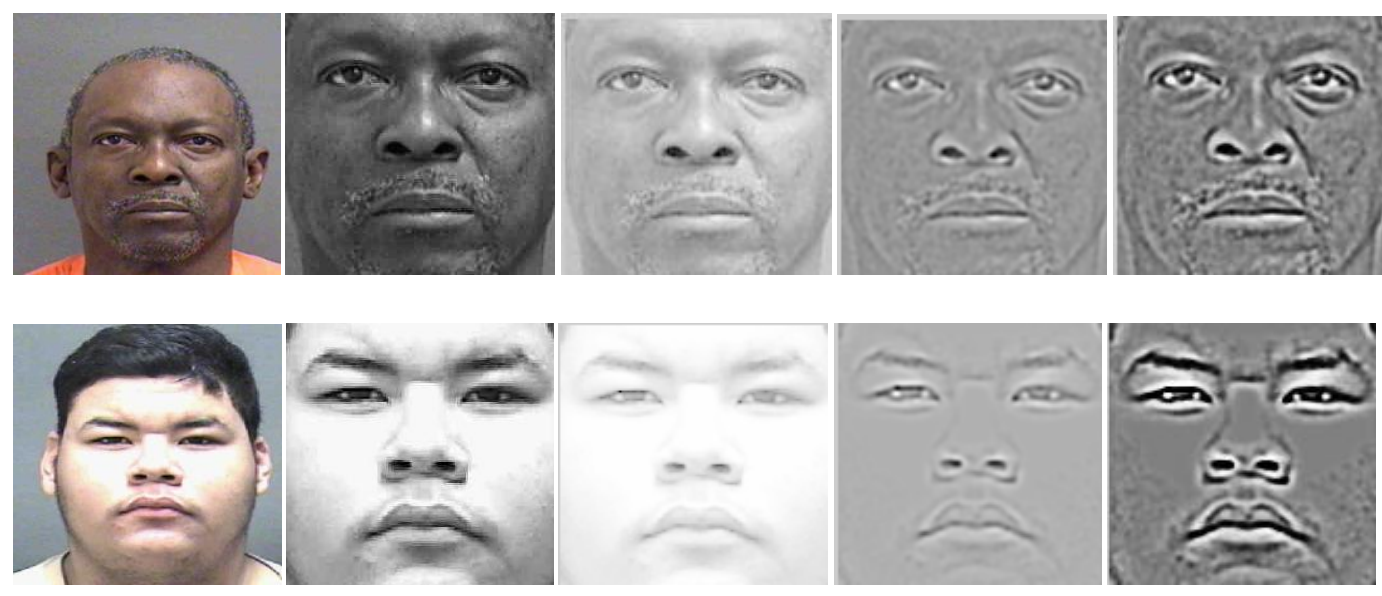

Fig. 3 Preprocessed MORPH images; Original image, face detection, Gamma correction, DoG filtering and normalization

After preprocessing, the preprocessed images are directly given to VGG16 model for feature extraction as well as classification. Based on the various convolution filters, the texture features are extracted and max-pooling layer is used as a dimension reduction technique. The performance of AGC system is measured in terms of accuracy. Table 2 shows the obtained accuracies by VGG16 system. From the randomly selected images, training of VGG16 model is made by $1 / 2$ of images and testing is made by the remaining images. It is inferred from the results that the average accuracy to classify age groups is over $90 \%$. 
TABLE 2 Performance of AGC system using VGG16 model

\begin{tabular}{|c|c|c|c|}
\hline \multirow{2}{*}{ Iteration } & \multicolumn{3}{|c|}{ Accuracy (\%) } \\
\cline { 2 - 4 } & Senior Adult & Adolescence & Adult \\
\hline 1 & 90.08 & 92.16 & 89.60 \\
\hline 2 & 89.28 & 92.08 & 90.56 \\
\hline 3 & 90.24 & 91.92 & 91.60 \\
\hline 4 & 90.40 & 91.84 & 90.24 \\
\hline 5 & 91.76 & 91.60 & 91.92 \\
\hline 6 & 91.60 & 91.68 & 89.76 \\
\hline 7 & 92.16 & 89.68 & 90.40 \\
\hline 8 & 90.24 & 89.52 & 91.68 \\
\hline 9 & 90.08 & 90.24 & 89.92 \\
\hline 10 & 89.28 & 90.88 & 92.08 \\
\hline Average & $\mathbf{9 0 . 5 1}$ & $\mathbf{9 1 . 1 6}$ & $\mathbf{9 0 . 7 7}$ \\
\hline
\end{tabular}

\section{CONCLUSION}

In this study, deep learning based AGC system using VGG16 architecture is presented. It classifies the face images in the MORPH database into their respective age groups such as senior adult, adolescence and adult. It uses a series of preprocessing stage which includes face detection, Gamma correction, DoG filtering and normalization to increase the accuracy of AGC system. The AGC system recognizes the age groups using deep learning approach with the help of VGG16 model. Results show that the AGC system provides over 90\% average recognition accuracy obtained by randomly selected (10 times) different testing samples.

\section{REFERENCES}

[1]. L. Liu, J. Liu, and J. Cheng, "Age-group classification of facial images", International Conference on Machine Learning and Applications, 2012, pp. 693-696.

[2]. A.K. Saxena, S. Sharma, and V.K. Chaurasia, "Human age-group estimation using curvelet features", International Conference on Advances in Computing and Communication Engineering, 2015, pp. 610-615.

[3]. S. Izadpanahi and Ö. Toygar, "Geometric feature based age classification using facial images", IET Conference on Image Processing, 2012, pp. 1-5.

[4]. S.H. Lee, and Y.M. Ro, "Local age group modeling in unconstrained face images for facial age classification", IEEE International Conference on Image Processing, 2014, pp. 1395-1399.

[5]. A. Asthana, and S.K. Singh, "Frontal facial images based age classify via SVM", International Conference on Electronics and Communication Systems, 2015, pp. 644-649.

[6]. J.B. Ko, W. Lee, S.E. Choi, and J. Kim, "A gender classification method using age information", International Conference on Electronics, Information and Communications, 2014, pp. 1-2.

[7]. K. Tonchev, I. Paliy, O. Boumbarov, and S. Sokolov, "Human age-group classification of facial images with subspace projection and support vector 
machines", IEEE International Conference on Intelligent Data Acquisition and Advanced Computing Systems, 2011, pp. 439-443.

[8]. J.H. Jeon, J.Y. Lee, J.S. Bae, and J.O. Kim, "Gradient local binary patterns for age group estimation", IEEE International Symposium on Consumer Electronics, 2014, pp. 1-2.

[9]. A. Yarlagadda, J.V.R. Murthy, and M.K. Prasad, "A novel method for human age group classification based on Correlation Fractal Dimension of facial edges", Journal of King Saud University-Computer and Information Sciences, Vol. 27, No. 4, 2015, pp. 468-476.

[10]. K. Ueki, T. Hayashida, and T. Kobayashi, "Subspace-based age-group classification using facial images under various lighting conditions", International Conference on Automatic Face and Gesture Recognition, 2017, pp. 6-pp.

[11]. W.B. Horng, C.P. Lee, and C.W. Chen, "Classification of age groups based on facial features", Tamkang Journal of Science and Engineering, Vol. 4, No. 3, pp. 183-192.

[12]. J. Jagtap, and M. Kokare, "Human age classification using facial skin analysis and multi-class support vector machine", International Conference on Signal and Information Processing, 2016, pp. 1-5.

[13]. M.A. Hajizadeh, and H. Ebrahimnezhad, "Classification of age groups from facial image using histograms of oriented gradients", Iranian Conference on Machine Vision and Image Processing, 2011, pp. 1-5.

[14]. M.R. Hosen, S. Hasan, M.M. Hasan, and R.K. Das, "Age classification based on EMG signal using Artificial Neural Network", International Conference on Electrical Engineering and Information Communication Technology, 2015, pp. 1-5.

[15]. J. Nithyashri, and G. Kulanthaivel, "Classifying the Human Age Using Discrete Wavelet Transform KNN Classifer and MORPH Database", Journal of Computer Applications, Vol. 6, No. 4, 2013, pp. 102-106.

[16]. K. Ricanek, and T. Tesafaye, "MORPH: A longitudinal Image Database of normal adult age - progression", International Conference on Automatic face and Gesture Recognition, 2006, pp. 341- 345. 